\title{
ON NONOSCILLATORY SOLUTIONS OF A NONLINEAR DIFFERENTIAL EQUATION
}

R. GRIMMER

\begin{abstract}
Sufficient conditions are given which insure that all nonoscillatory solutions of $\left(p(t) x^{\prime}\right)^{\prime}+h(x) x^{\prime}+q(t) g(x)=f(t)$ tend to zero as $t$ tends to infinity.
\end{abstract}

In this paper we examine the behavior of the nonoscillatory solutions of the equation

$$
\left(p(t) x^{\prime}\right)^{\prime}+h(x) x^{\prime}+q(t) g(x)=f(t)
$$

where $p, q$, and $f$ are real valued and continuous for $t \geqq 0$ and $h$ and $g$ are real valued and continuous for all real $x$. Also, we assume $p(t)$ and $q(t)$ are positive for all $t, x g(x)>0$ for $x \neq 0$, and $x H(x) \geqq 0$ where $H(x)$ is defined by

$$
H(x)=\int_{0}^{x} h(u) d u .
$$

In a recent paper [1], Hammett considered the equation

$$
\left(p(t) x^{\prime}\right)^{\prime}+q(t) g(x)=f(t)
$$

with $p, q, g$, and $f$ as above and gave conditions which insure that every nonoscillatory solution $x(t)$ of (2) tends to zero as $t \rightarrow \infty$. The purpose of this paper is to prove a similar result for (1) which when applied to (2) extends Hammett's result.

As an example of the possible nonoscillatory behavior of solutions of (2) we note that $(2+\sin t) / t$ is a nonoscillatory solution of an equation of the form

$$
\left(t x^{\prime}\right)^{\prime}+x^{3}=f(t)
$$

with $f(t)=f_{1}(t)-(\cos t) / t-\sin t$ where $\int^{\infty}\left|f_{1}(t)\right| d t<\infty$. Our main result will show that all nonoscillatory solutions of (3) tend to zero as $t \rightarrow \infty$ while Hammett's result does not apply as he requires $\int^{\infty}|f(t)| d t<\infty$.

Received by the editors October $15,1971$.

AMS 1969 subject classifications. Primary 3442, 3450; Secondary 3453.

Key words and phrases. Nonoscillatory, oscillatory, nonlinear, differential equation, solution, approach zero.

(c) American Mathematical Society 1972 
THEOREM 1. Suppose the following conditions are valid:

(i) $p(t)>k>0, k$ constant,

(ii) $\int^{\infty}(1 / p(t)) d t=\infty$,

(iii) $|g(x)|$ is bounded away from zero if $|x|$ is bounded away from zero.

(iv) if $\left\{t_{n}\right\}$ and $\left\{s_{n}\right\}$ are sequences with $0<t_{n}<s_{n}<t_{n+1}$ and $s_{n}-t_{n}>\varepsilon$ where $\varepsilon$ is a positive constant, then

$$
\sum_{n=0}^{\infty} \int_{t_{n}}^{s_{n}} q(t) d t=\infty,
$$

(v) $F(t)=\int_{0}^{t} f(u) d u$ is bounded.

Then if $x(t)$ is a nonoscillatory solution of (1), $x(t) \rightarrow 0$ as $t \rightarrow \infty$.

Proof. Let $x(t)$ be a nonoscillatory solution of (1) and consider the equation

$$
x^{\prime}=(y-H(x)+F(t)) / p(t), \quad y^{\prime}=-q(t) g(x),
$$

which is equivalent to (1). Corresponding to $x(t)$ we consider the solution $(x(t), y(t))$ of $(4)$ and assume that $x(t)$ is eventually positive. The argument if $x(t)$ is eventually negative is similar and will be omitted.

We note first that

$$
\liminf _{t \rightarrow \infty} x(t)=0 .
$$

If this is not the case, then there exists $T>0$ such that $x(t)$ and $g(x(t))$ are bounded below by positive constants for $t \geqq T$. From (4) it follows that

$$
y(t)-y(T)=-\int_{T}^{t} q(s) g(x(s)) d s \rightarrow-\infty .
$$

Thus, for $t \geqq T_{1}>T$ for some $T_{1}$ sufficiently large,

$$
x^{\prime}(t) \leqq(y(t)+F(t)) / p(t) \leqq-(1 / p(t))
$$

as $F(t)$ is bounded and $x H(x) \geqq 0$. This implies that $x(t) \rightarrow-\infty$ as $t \rightarrow \infty$ which further implies $x(t)$ has a zero after $T_{1}$. This, however, is a contradiction and (5) follows.

From the above argument, we see also that we must have

$$
\int^{\infty} q(t) g(x(t)) d t<\infty
$$

Now it follows from (4) that $y^{\prime}(t) \leqq 0$ if $x(t) \geqq 0$ and so $y(t)$ is bounded above as $t \rightarrow \infty$. Thus, $x^{\prime}(t) \leqq K / p(t)$ for some $K>0$ and as $p(t)>R>0$, $x^{\prime}(t)$ is bounded above as $t \rightarrow \infty$. If

$$
\lim _{t \rightarrow \infty} \sup x(t)=N>0
$$


then there are sequences $t_{n}$ and $s_{n}$ with $t_{n}<s_{n}<t_{n+1}$ such that $x(t)>0$ for $t \geqq t_{0}, x\left(t_{n}\right)=N / 4, x\left(s_{n}\right)=3 N / 4$, and $N / 4 \leqq x(t) \leqq 3 N / 4$ for $t_{n} \leqq t \leqq s_{n}$. As $x^{\prime}(t)$ is bounded above, there is an $\varepsilon>0$ such that $s_{n}-t_{n} \geqq \varepsilon$ and so

$$
\int_{t_{0}}^{s_{n}} q(t) g(x(t)) d t \geqq M \sum_{j=0}^{n} \int_{t_{j}}^{s_{j}} q(t) d t
$$

where $M>0$ is chosen so that $g(x)>M$ when $N / 2 \leqq x \leqq 3 N / 4$. It follows now from (iv), howver, that $\int_{0}^{\infty} q(t) g(x(t)) d t=\infty$. This contradiction of (6) completes the proof.

Consider now equation (2) and the Liouville transformation given by $s=\int(1 / p(u)) d u, z(s)=x(t)$. Then $z$ satisfies the equation

$$
\ddot{z}+p(t) q(t) g(z)=p(t) f(t) \quad(\cdot=d / d s)
$$

and $z(s)$ is nonoscillatory if and only if $x(t)$ is nonoscillatory. Further, if (ii) is valid, $z(s) \rightarrow 0$ as $s \rightarrow \infty$ if and only if $x(t) \rightarrow 0$ as $t \rightarrow \infty$. Thus we may determine the behavior of the nonoscillatory solutions of (2) by examining the nonoscillatory solutions of (7). These considerations lead to the following corollary which generalizes the main result of Hammett [1] who required that both $p(t)$ and $q(t)$ be bounded below by a positive constant and that $f(t)$ be absolutely integrable on $[0, \infty)$.

COROLlary 2. Assume that (ii), (iii) and (v) are valid and that $p(t) q(t)$ is bounded below by a positive constant. Then every nonoscillatory solution $x(t)$ of (2) tends to zero as $t \rightarrow \infty$.

Proof. From the above discussion, it follows that we need only show that every nonoscillatory solution of (7) tends to zero as $s \rightarrow \infty$. This follows immediately from Theorem 1 , however, upon noting that

$$
\int_{0}^{s} p(t) f(t) d v=\int_{0}^{t} f(u) d u
$$

where $t=t(s)$ is the inverse function of $s=s(t)$.

\section{REFERENCE}

1. Michael E. Hammett, Nonoscillation properties of a nonlinear differential equation, Proc. Amer. Math. Soc. 30 (1971), 92-96.

Department of Mathematics, Southern Illinois University, Carbondale, ILLINOIS 62901 